Please do not remove this page

RMIT

UNIVERSITY

\title{
Nanostructure Evolution of Biomimetic Hydrogel from Silk Fibroin and Poly(N-Vinylcaprolactam): A Small Angle Neutron Scattering Study
}

Balu, Rajkamal; Whittaker, Jasmin; Mata, Jitendra; Dutta, Naba

https://researchrepository.rmit.edu.au/esploro/outputs/9921863571901341/filesAndLinks?institution=61RMIT_INST\&index=null

Balu, R., Whittaker, J., Mata, J., \& Dutta, N. (2018). Nanostructure Evolution of Biomimetic Hydrogel from Silk Fibroin and Poly(N-Vinylcaprolactam): A Small Angle Neutron Scattering Study. In Gels and Other Soft Amorphous Solids (pp. 71-89). American Chemical Society.

https://researchrepository.rmit.edu.au/discovery/fulldisplay/alma9921863571901341/61RMIT_INST:Resea rchRepository

Document Version: Accepted Manuscript

Repository homepage: https://researchrepository.rmit.edu.au

(c) 2018 American Chemical Society

Downloaded On 2023/04/26 22:01:50 +1000 


\title{
Nanostructure Evolution of Biomimetic Hydrogel from silk fibroin and poly(N-vinylcaprolactam): A Small Angle Neutron Scattering study
}

\author{
Rajkamal Balu, ${ }^{1}$ Jasmin Whittaker, ${ }^{2}$ Jitendra P. Mata, ${ }^{3}$ Naba K. Dutta, ${ }^{* 1,2}$ and \\ Namita Roy Choudhury*,1,2 \\ ${ }^{1}$ School of Engineering, RMIT University, Melbourne, VIC 3000, Australia \\ ${ }^{2}$ Future Industries Institute, University of South Australia, Mawson Lakes, SA 5095, Australia \\ ${ }^{3}$ Australian Centre for Neutron Scattering, Australian Nuclear Science and Technology Organisation, \\ Lucas Heights, NSW 2234, Australia
}

*Corresponding Author E-mail: naba.dutta@,rmit.edu.au; namita.choudhury@rmit.edu.au 


\begin{abstract}
The nanostructure of biomimetic hydrogels fabricated using regenerated silk fibroin (RSF) protein and thermoresponsive poly(N-vinylcaprolactam) (PVCL) polymer via physical and chemical crosslinking methods have been studied using Small Angle Neutron Scattering (SANS) technique. RSF showed no gelation and/or secondary structure (Gaussian coil) change at temperatures between $12{ }^{\circ} \mathrm{C}$ and $35^{\circ} \mathrm{C}$, whereas PVCL formed physical hydrogels by molecular self assembly exhibiting swollen coil to Gaussian coil structure change above a lower critical solution temperature (LCST) of $\sim 32{ }^{\circ} \mathrm{C}$. RSF-PVCL blend solutions were also observed to form physical hydrogels above this LCST. However, RSF-PVCL hybrid system exhibited swollen coil to collapsed coil structure change, suggesting relatively increased gelation compared to PVCL. Contrast variation SANS experiments demonstrate that in RSF-PVCL hybrid system, RSF provokes the intrinsic structural changes in PVCL without undergoing any structural change of its own. Unlike PVCL and other biomimetic polymer hydrogels, RSF hydrogels exhibited ordered structures containing hydrophobic domains which exhibit sharp interfaces with hydrophilic domains and/or amorphous protein matrix. Further, photochemically crosslinked RSF and RSF-PVCL hybrid hydrogels showed variation in the order of secondary structures in hydrophilic domains, which can be associated to their reported difference in water uptake behavior.
\end{abstract}

\title{
Introduction
}

Biomimetic hydrogels are three-dimensional macromolecular network structures formed by physical crosslinking (hydrogen bonding, ionic or hydrophobic interactions) or chemical crosslinking (covalent bonding) of synthetic polymers and/or natural proteins that are biocompatible in nature. Biomimetic hydrogels mimic biological extracellular matrix structures and absorb large amount of water without disintegrating (1). Notable examples of biomimetic hydrogels include: poly(acrylic acid), poly(ethylene oxide), poly(Nvinylcaprolactam), collagen, alginate and silk fibroin (1-7). In particular, hydrogels of poly(N-vinylcaprolactam) (PVCL) and silk fibroin have been extensively studied in the field 
of biomedical and tissue engineering $(8,9)$. PVCL is a nonionic thermoresponsive synthetic polymer widely synthesized by free radical polymerization, which exhibits lower critical solution temperature (LCST) characteristics in the physiological temperature range $(\sim 32-35$ $\left.{ }^{\circ} \mathrm{C}\right)(8)$. Though PVCL is widely applied in drug delivery and cosmetics, it is a relatively soft material, which limits its applications in tissue engineering (10). On the other hand, silk fibroin is a fibrous natural protein produced by silk worms such as Bombyx mori and can be extracted from their cocoons by chemical regeneration methods (11). The extracted silk fibroin, commonly known as regenerated silk fibroin (RSF), has been widely applied in tissue engineering for its high mechanical strength, excellent biocompatibility, controlled biodegradability and ability to support cellular interactions (12). Unlike PVCL and other thermoresponsive proteins (eg. Resilin), RSF does not show any critical solution temperature characteristics, which limits its applications in drug delivery (13-15). Developing hydrogels that exhibit combined properties of stimuli responsiveness and mechanical properties is of significant interest in the biomedical field. It has been reported that RSF and PVCL form stable hydrogen bonding interactions, which have been applied to fabricate capsules and coatings $(16,17)$. Recently, we reported a facile fabrication of RSF-PVCL hybrid hydrogels by a photochemical crosslinking method (18). However, the effect of RSF on the phase behaviour of PVCL and their resultant hybrid hydrogel nanostructure has not been studied. Fundamental understanding of the nanostructure of RSF-PVCL hybrid hydrogel is crucial to optimize their physicochemical and biological properties for desired applications. In this chapter, we study the intrinsic structure and assembly of RSF and PVCL molecules in both physically and chemically crosslinked RSF-PVCL hybrid hydrogels using small angle neutron scattering (SANS) technique.

SANS is an experimental technique which uses elastic neutron scattering at small scattering angles to provide structural information of various nanomaterials at a length scale 
of about 1-1000 nm (19). SANS is complimentary to small angle X-ray scattering (SAXS) and provides information from both bulk and surfaces/interfaces of materials which is not possible with other techniques (19). Thefore, SANS is advantageous in studying the structure and assembly of macromolecules in concentrated solutions and gel phases (20-21). Moreover, SANS/SAXS is the only technique which provides nano to microstructure information of hydrogels in their native and swollen state without compromising structures by sample preparation that are common in other techniques such as electron microscopy and atomic force microscopy (19). Advantages of SANS over SAXS include its sensitivity to lighter elements such as hydrogen and the possibility of isotope labeling (22). In particular, the unique interaction of neutrons with hydrogen and its isotope deuterium provides contrastvariation capability, where water $\left(\mathrm{H}_{2} \mathrm{O}\right)$, deuterium oxide $\left(\mathrm{D}_{2} \mathrm{O}\right)$ and their mixtures can be used as solvent/background medium to contrast match one component and study the structure of other in a multi-component system such as RSF-PVCL hybrid hydrogels (22).

\section{Experimental}

\section{Materials}

Bombyx mori silk fibers were purchased from Beautiful Silks, Australia. N-vinylcaprolactam (VCL), 2,2'-azoisobutyronitrile (AIBN) and Tris(2,2'-bipyridyl)dichlororuthenium(II) hexahydrate $\left(\operatorname{Ru}(\mathrm{bpy})_{3}\right)$ were purchased from Sigma Aldrich, Australia. Dialysis tube and syringe filters were purchased from ThermoScientific, Australia. $\mathrm{D}_{2} \mathrm{O}$ was supplied by the Australian Nuclear Science and Technology Organisation (ANSTO). All other chemicals were purchased from Chem-Suppy, Australia.

\section{Preperation of RSF solution}

For preparation of RSF aqueous solution, the silk fibers were first degummed by boiling in $0.5 \mathrm{~N}$ sodium carbonate. The degummed fibers were then washed with water, air-dried and 
dissolved in calcium chloride-ethanol-water mixture $\left(1: 8: 2\right.$ mole ratio) at $70{ }^{\circ} \mathrm{C}$ for $3 \mathrm{~h}(7)$. The dissolved solution was then centrifuged at $10,000 \mathrm{rpm}$ for $30 \mathrm{~min}$ to remove any precipitates, dialysed (using 3.5 MWCO dialysis tube) against $\mathrm{D}_{2} \mathrm{O}$ for 4 days, and filtered using $1.2 \mu \mathrm{m}$ syringe filter. The molecular weight of prepared RSF was measured to be approximately 30 to $200 \mathrm{kDa}$ using gel electrophoresis (7).

\section{Synthesis of PVCL}

PVCL was prepared via free radical polymerisation of the VCL monomer with AIBN as the initiator and toluene as the solvent at $70{ }^{\circ} \mathrm{C}$ for $24 \mathrm{~h}$ under nitrogen atmosphere (18). The polymer was recovered through precipitation in diethyl ether and purified by dissolving in a mixture of acetone and water $(3: 7)$ followed by precipitation by heating the solution to $80{ }^{\circ} \mathrm{C}$ and drying in vacuum at $50{ }^{\circ} \mathrm{C}$ for $3 \mathrm{~h}$ (18). The molecular weight of synthesized PVCL was measured to be $\sim 35.4 \mathrm{kDa}$ using viscosity measurements (18).

\section{Fabrication of RSF-PVCL blend solutions and hybrid hydrogels}

For RSF-PVCL blend solution preparation, a predetermined amount of aqueous solution of PVCL dissolved in $\mathrm{D}_{2} \mathrm{O}$ was added slowly into RSF solution under gentle stirring followed by sonication for several minutes to allow adequate distribution of molecules. For physically crosslinked RSF-PVCL hybrid hydrogel fabrication, solution blends of RSF and PVCL was equilibrated at $35{ }^{\circ} \mathrm{C}$ for $1 \mathrm{hr}$. For chemically crosslinked RSF-PVCL hybrid hydrogel fabrication, predefined amount of PVCL was first mixed with RSF solution followed by the photo-catalyst $\mathrm{Ru}(\mathrm{bpy})_{3}$ and the electron acceptor ammonium persulfate. The mixed solution was then poured into Teflon moulds and exposed to a $250 \mathrm{~W}$ white light for 2 minutes. The gels formed were then turned over and exposed for a further 30 seconds to ensure complete crosslinking (18). The excess reactants from crosslinked hydrogels were removed by dialysing against ultra-pure water and vacuum dried. 


\section{Small angle neutron scattering}

SANS analysis was performed on RSF, PVCL and PVCL-RSF solution blends and crosslinked hydrogels using the ANSTO Quokka SANS instrument (23). The scattering profiles of samples were recorded against the scattering vector, $q$ (equation 1) in the range of $0.006-0.36 \AA^{-1}(24)$.

$$
q=\frac{4 \pi \sin \theta}{\lambda}
$$

where $2 \theta$ is the angle of scattering and $\lambda$ is the wavelength of the neutron beam ( $5 \AA$ ). Source to sample aperture distances of $2 \mathrm{~m}$ and $8 \mathrm{~m}$ were employed to cover the $q$ range. The solutions prepared in $\mathrm{D}_{2} \mathrm{O}$ and hydrogels equilibrium swollen in $\mathrm{D}_{2} \mathrm{O}$ (cut into discs of ca. 15 $\mathrm{mm}$ ) were loaded into demountable Quokka cell assembly of $20 \mathrm{~mm}$ diameter and $2 \mathrm{~mm}$ path length along with excess $\mathrm{D}_{2} \mathrm{O}$. The neutron scattering length density (SLD) of $\mathrm{D}_{2} \mathrm{O}(6.36 \times$ $\left.10^{-6} \AA^{-2}\right)$ provides good contrast against RSF $\left(\sim 3.2 \times 10^{-6} \AA^{-2}\right)$, PVCL $\left(\sim 1.1 \times 10^{-6} \AA^{-2}\right)$, and also limits the incoherent background scattering from hydrogen in the system $(25,26)$. For contrast matching SLD of PVCL, the gels were equilibrium swollen in $\mathrm{D}_{2} \mathrm{O} / \mathrm{H}_{2} \mathrm{O}$ mixture $\left(22 \% \mathrm{D}_{2} \mathrm{O}\right)$ prior to loading in Quokka cells with excess of respective medium. All measurements were performed with a sample aperture diameter of $12.5 \mathrm{~mm}$, and the obtained data were reduced using NCNR SANS reduction macros (modified for the QUOKKA instrument) using the Igor software package with data corrected (considering detector sensitivity) for empty cell and cadmium scattering (27). Further, the data were transformed to absolute scale using an attenuated direct beam transmission measurement. The solvent/medium scattering were subtracted from the respective sample data using the PRIMUS computer program (28). The incoherent background scattering was determined with a power law fit at the very high- $q$ region using the SasView computer program (http://www.sasview.org/) and the constant value was subtracted from the respective sample data for analysis (29). The structural parameters of the fabricated hydrogels were determined 
by fitting the neutron scattering data with different functions/models using the SasView computer program.

\section{Results and discussion}

\section{Intrinsic structure of RSF, PVCL and their blend solutions as a function of temperature}

In small angle scattering (SAS), the overall neutron scattering intensity, I(q) of polymer solutions are generally analysed by a function of both the form factor, $\mathrm{P}(\mathrm{q})$ which is an intrascattering event and the structure factor, $\mathrm{S}(\mathrm{q})$ which is an inter-scattering event, as given in equation 2 (19).

$$
I(q)=\varphi P(q) S(q) \ldots(2)
$$

where $\varphi$ is a scaling factor related to the difference in SLD and the volume of scatterers. The intra-molecular scattering (high-q and mid-q) contains information about the distribution of inter-atomic distances within a molecule and can be interpreted in terms of its size and shape, whereas the inter-molecular scattering (low-q) provides information about the orientationaveraged polymer-polymer interactions and can be interpreted in terms of aggregate size or spatial distance (30). At low concentrations of protein and polymer solutions, the scattering data is highly dominated by the intra-molecular events, i.e. $S(q)=1(24)$. Figure 1A, 1B and 1C shows the double logarithmic SANS intensity profile of RSF, PVCL and their solution blends, respectively at a low concentration of $2 \mathrm{wt} \%$. The curves show two distinctive regions: a high-q Porod region $\left(0.03<\mathrm{q}<0.36 \AA^{-1}\right)$ and a mid-q Guinier region $(0.006<\mathrm{q}<$ $\left.0.03 \AA^{-1}\right)$. In general, the small angle scattering intensity probing to the local structure of tested system can be determined from Porod slope (n) measured from the Porod region (highq) which yields information about the fractal dimension of the scattering objects, and the Guinier approximation at the Guinier region (mid-q) which allows for estimation of the radius-of-gyration ( $\mathrm{Rg}$ ) of scattering object (30). The scattering pattern of RSF was observed 
to show no noticeable difference across the tested temperature range of $12{ }^{\circ} \mathrm{C}$ to $35^{\circ} \mathrm{C}$, which suggests the RSF to be non thermoresponsive at tested temperatures. Conversely, PVCL and RSF-PVCL blend solutions showed increase in scattering intensity and Porod slope with increase in temperature, which supports the thermoresponsive property of PVCL reported in literature (31). The Porod slope was obtained from the high-q scattering data through a powerlaw fit using SasView computer program. The estimated Porod slopes of samples are given in Table 1.

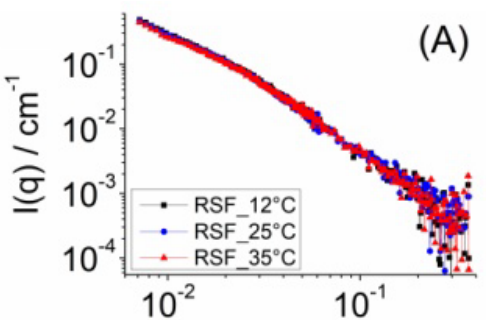

$\mathrm{q} / \AA^{-1}$

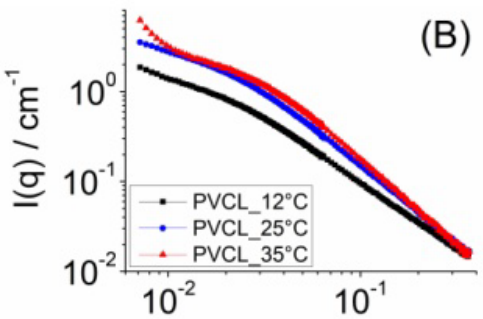

$\mathrm{q} / \AA^{-1}$

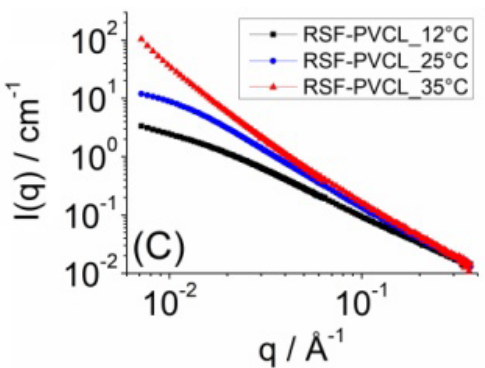

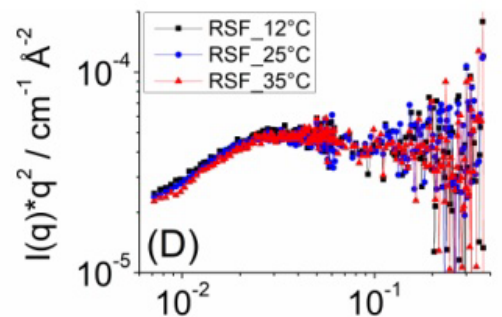

$\mathrm{q} / \AA^{-1}$
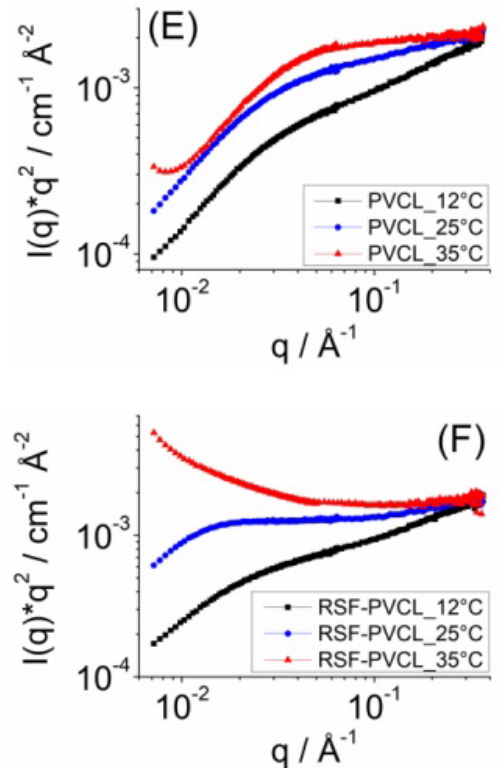

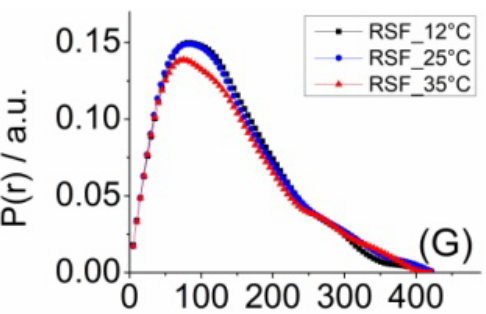

$r / \AA$
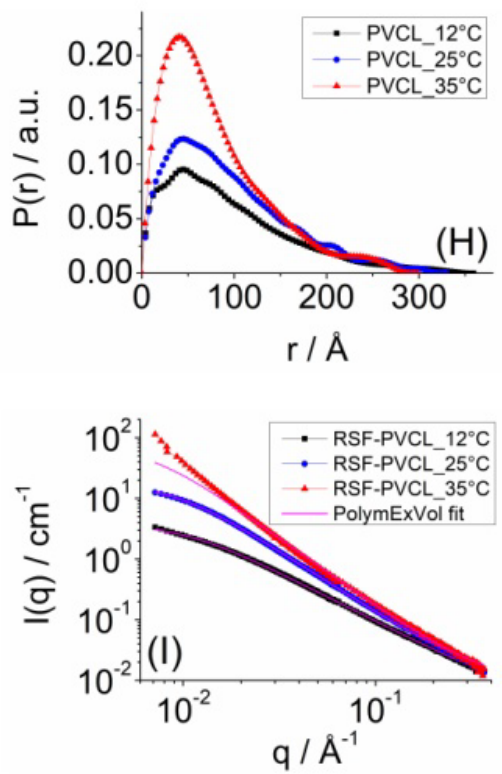

Figure 1. (A), (B), (C) are SANS curves, and (D), (E), (F) are respective Kratky plots of RSF, PVCL and RSF-PVCL solution blend as a function of temperature. $(\mathrm{G})$ and $(\mathrm{H})$ are distance distribution functions of RSF and PVCL, respectively as a function of temperature. (I) is the polymer excluded volume model fit of RSF-PVCL solution blend as a function of temperature. 
The RSF exhibited a Porod slope of $\sim 2$ at all tested temperatures, which is a signature of Gaussian coil in dilute environment and suggests the RSF structure to be largely unordered in solution (32). On the other hand, the PVCL exhibited a Porod slope of $1.56 \pm 0.02$ at $12{ }^{\circ} \mathrm{C}$, which increased to $1.82 \pm 0.02$ at $25^{\circ} \mathrm{C}$ and $2.00 \pm 0.02$ at $35{ }^{\circ} \mathrm{C}$ subsequently, suggesting changes in the intrinsic structure of PVCL molecules with temperature. In general, a Porod slope of $\sim 1$ corresponds to a rigid rod like structure, $\sim 5 / 3$ for a fully swollen coil, $\sim 2$ for Gaussian coil and between 2 and 3 for "mass fractals" such as branched systems (gels) or networks (33). Therefore, PVCL exhibiting swollen coil to Gaussian coil structure change occurs above a lower critical solution temperature (LCST) of $\sim 32{ }^{\circ} \mathrm{C}$. Moreover, the PVCL solution was observed to turn turbid at $35^{\circ} \mathrm{C}$ and exhibits slight upturn at low-q scattering suggesting molecular self assembly, aggregation or occurance of gelation in the system. Interestingly, the RSF-PVCL blend solutions exhibited a Porod slope of $1.72 \pm 0.02$ at $12{ }^{\circ} \mathrm{C}$, which increased to $2.0 \pm 0.02$ at $25{ }^{\circ} \mathrm{C}$ and $2.27 \pm 0.02$ at $35{ }^{\circ} \mathrm{C}$, suggesting $\mathrm{RSF}$ induced/enhanced further structural changes (swollen coil to collapsed coil) in the blend system with temperature. Further, the turbidity of solution was observed to be more prominent for RSF-PVCL blend system at $35{ }^{\circ} \mathrm{C}$ with a strong scattering upturn observed at low-q suggesting increased gelation compared to PVCL. This structural change could also be associated with RSF induced tuning of LCST of PVCL, reported previously (13); where the onset of LCST of PVCL decreased from $\sim 32{ }^{\circ} \mathrm{C}$ to $\sim 25{ }^{\circ} \mathrm{C}$ in the presence of RSF. The structural change can be attributed to strong hydrogen bonding interactions between RSF and PVCL $(16,17)$. Further, the unfolded-ness of RSF and PVCL was qualitatively assessed by means of Kratky plot (Figure 1D, 1E and 1F). The Kratky plot divides-out the decay of the scattering and clearly shows any structural differences in the sample (33). The Kratky plot of RSF and PVCL displayed initial monotonic increase in mid-q followed by a plateau or slight increase in high-q, which is characteristic of an overall random coil secondary structural 
conformation of macromolecules in solution (33). However, at $35{ }^{\circ} \mathrm{C}$, PVCL showed increased scattering intensity at low-q region, which further confirms the existence of larger structural assembly in the system. The RSF-PVCL blends showed scattering trend similar to that of PVCL at $12{ }^{\circ} \mathrm{C}$ and $25{ }^{\circ} \mathrm{C}$, however, with a well pronounced feature in mid-q region, which further establishes larger structural assembly of molecules in the system leading to coaccervation or gelation.

In SAS data analysis, Guinier's approximation is commonly used for estimation of particle/molecular size such as radius-of-gyration $(\mathrm{Rg})$. However, the approximation is very sensitive to scattering intensities at small q-values and has been reported to underestimate the $\mathrm{Rg}$ for structurally unordered protein and polymer coils (34). On the other hand, the pairdistance distribution function, $\mathrm{P}(\mathrm{r})$ is a model independent function that calculates the $\mathrm{Rg}$ by inverse Fourier transform of the entire scattering spectrum with a histogram of all of the interatomic distances (r). Therefore, here we estimated the Rg of RSF and PVCL systems using $\mathrm{P}(\mathrm{r})$ function (Figure $1 \mathrm{G}$ and $1 \mathrm{H})(30,35)$. However, for RSF-PVCL blend system that showed mass fractal Porod exponent at $35{ }^{\circ} \mathrm{C}$, the $\mathrm{Rg}$ was estimated using polymer excluded volume model fit to scattering data using SasView computer program (Figure 1I). The polymer excluded volume model describes the scattering from polymer chains subject to excluded volume effects and has been used as a template for describing mass fractals or collapsed polymer chains, therefore chosen for estimating Rg of RSF-PVCL blend system (36). The form factor, $P(q)$ is presented in the following integral form (37):

$$
P(q)=2 \int_{0}^{1} d x(1-x) \exp \left[-\frac{q^{2} a^{2}}{6} n^{2 v} x^{2 v}\right]
$$

where $v$ is the excluded volume parameter (which is related to the Porod exponent/slope $n$ as $v=1 / \mathrm{n})$, a is the statistical segment length of the polymer chain, and $\mathrm{n}$ is the degree of polymerization (37). The estimated structural parameters of samples are given in Table 1 . The $\operatorname{Rg}$ of RSF ( $\sim 11 \mathrm{~nm})$ was observed to be consistent between tested temperatures of $12{ }^{\circ} \mathrm{C}$ and 
$35{ }^{\circ} \mathrm{C}$, whereas the Rg of PVCL was observed to decrease with increase in temperature. The decrease in Rg along with increase in Porod slope suggests occurrance of change in structure of PVCL in the molecular level leading to self assembly and aggregation. On the other hand, in RSF-PVCL blend system, with increase in temperature, the Rg was observed to increase along with Porod slope. This is due to the mid-q scattering of RSF-PVCL blend system observed to be dominated by low-q scattering with increase in temperature. At $35{ }^{\circ} \mathrm{C}$, the RSF-PVCL blend system showed Rg of $23.09 \pm 0.06$. A strong upturn at low-q intensity observed towards low-q suggests presence of structural co-assemblies on a range of length scales in the sample, extending beyond the measured SANS range $(>100 \mathrm{~nm})$.

Table 1. Structural parameters of solution samples estimated from SANS data.

\begin{tabular}{|c|c|c|c|c|c|c|}
\hline \multirow[t]{2}{*}{ Sample } & \multirow{2}{*}{$\begin{array}{c}\text { Temperature } \\
\left({ }^{\circ} \mathrm{C}\right)\end{array}$} & \multirow{2}{*}{\begin{tabular}{|l|} 
Power law fit \\
Porod slope
\end{tabular}} & \multirow{2}{*}{$\begin{array}{c}P(r) \\
\text { function } \\
\text { fit } \\
R_{g}(\mathrm{~nm})\end{array}$} & \multirow{2}{*}{$\begin{array}{c}\text { Polymer } \\
\text { excluded } \\
\text { volume fit } \\
R_{g}(n m)\end{array}$} & \multicolumn{2}{|c|}{ Guinier-Porod fit } \\
\hline & & & & & $R_{g}(\mathrm{~nm})$ & Porod slope \\
\hline \multirow[t]{6}{*}{ RSF } & 12 & $2.00 \pm 0.02$ & $11.30 \pm$ & - & $10.39 \pm 0.07$ & $2.00 \pm 0.10$ \\
\hline & & & 0.35 & & & \\
\hline & 25 & $2.00 \pm 0.02$ & $11.00 \pm$ & - & $10.34 \pm 0.07$ & $2.00 \pm 0.10$ \\
\hline & & & 0.35 & & & \\
\hline & 35 & $2.00 \pm 0.02$ & $11.03 \pm$ & - & $10.35 \pm 0.07$ & $2.00 \pm 0.10$ \\
\hline & & & 0.33 & & & \\
\hline \multirow[t]{6}{*}{ PVCL } & 12 & $1.56 \pm 0.02$ & $8.25 \pm$ & - & $5.39 \pm 0.01$ & $1.56 \pm 0.01$ \\
\hline & & & 0.16 & & & \\
\hline & 25 & $1.82 \pm 0.02$ & $8.07 \pm$ & - & $5.24 \pm 0.01$ & $1.82 \pm 0.01$ \\
\hline & & & 0.21 & & & \\
\hline & 35 & $2.00 \pm 0.02$ & $6.89 \pm$ & - & $4.29 \pm 0.01$ & $2.00 \pm 0.01$ \\
\hline & & & 0.29 & & & \\
\hline RSF- & 12 & $1.72 \pm 0.02$ & - & $13.01 \pm 0.06$ & $7.65 \pm 0.01$ & $1.72 \pm 0.01$ \\
\hline \multirow[t]{2}{*}{ PVCL } & 25 & $2.00 \pm 0.02$ & - & $16.48 \pm 0.04$ & $10.77 \pm 0.01$ & $2.00 \pm 0.01$ \\
\hline & 35 & $2.27 \pm 0.02$ & - & $23.09 \pm 0.06$ & $25.78 \pm 0.01$ & $2.27 \pm 0.01$ \\
\hline
\end{tabular}


To further validate and assess the reliability of estimated Porod exponent and $\mathrm{Rg}$, Guinier-Porod model was fit to SANS data of prepared macromolecules (Figure 2) (38). Guinier-Porod model is a shape-independent model which calculates scattering for a generalized Guinier/power law object and can be used to determine the size and dimensionality of scattering objects (38). Considering the structure of macromolecules in solution to be three-dimensional (3D), a dimension variable of zero (used for 3D objects) was used to obtain the structural parameters Porod exponent and $\mathrm{Rg}$ (38), and given in Table 1. The Porod exponent of samples obtained using Guinier-Porod model fit was observed to be consistent with values obtained using power law fit. The Rg of RSF obtained using GuinierPorod model fit was also in good aggrement with values obtained using $\mathrm{P}(\mathrm{r})$ function fit suggesting the prepared RSF to be in monomeric form at a concentration of $2 \mathrm{wt} \%$. However, the Rg of PVCL and RSF-PVCL blend solution obtained using Guinier-Porod model fit was observed to be less than that obtained using $\mathrm{P}(\mathrm{r})$ function fit, which suggests possibility of inter-molecular interaction in PVCL and RSF-PVCL blend solutions that may have contributed to higher Rg estimated using P(r) function fit. 

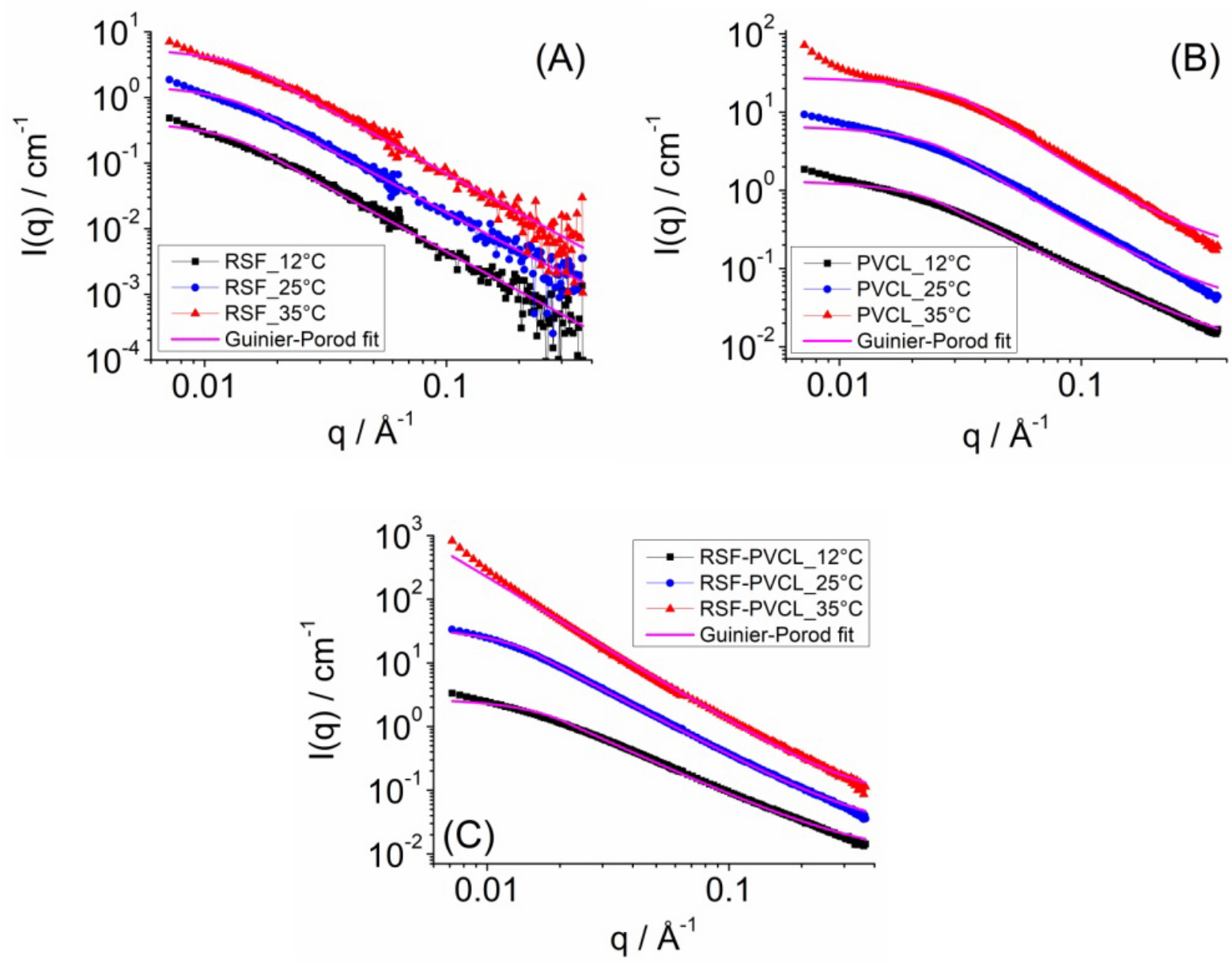

Figure 2. Guinier-Porod model fit to SANS data of (A) RSF, (B) PVCL and (C) RSF-PVCL blend system as a function of temperature. The individual data curves have been transposed for clarity.

In order to obtain an in-depth understanding of the intrinsic structure of RSF in the RSF-PVCL blend solution system, experiments were performed by contrast matching neutron SLD of PVCL (masking PVCL scattering). Figure 3 shows the PVCL contrast matched SANS intensity profile of RSF-PVCL blend system. The contrast matched data showed that the scattering intensity profile of RSF-PVCL blend system did not change with temperature, which indicates intrinsic structure of RSF in the RSF-PVCL blend solution system to be unaffected by the presence of PVCL and temperature. The RSF in RSF-PVCL blend system exhibited a Porod slope of $\sim 2$ (Gaussian coil) (33). This is also consistent with Kratky plot 
showing initial monotonic increase in mid-q followed by a plateau or slight increase in high-q (33). Therefore, in RSF-PVCL blend system it is evident that the observed structural change is dominated by change in intrinsic structure of PVCL rather than RSF.
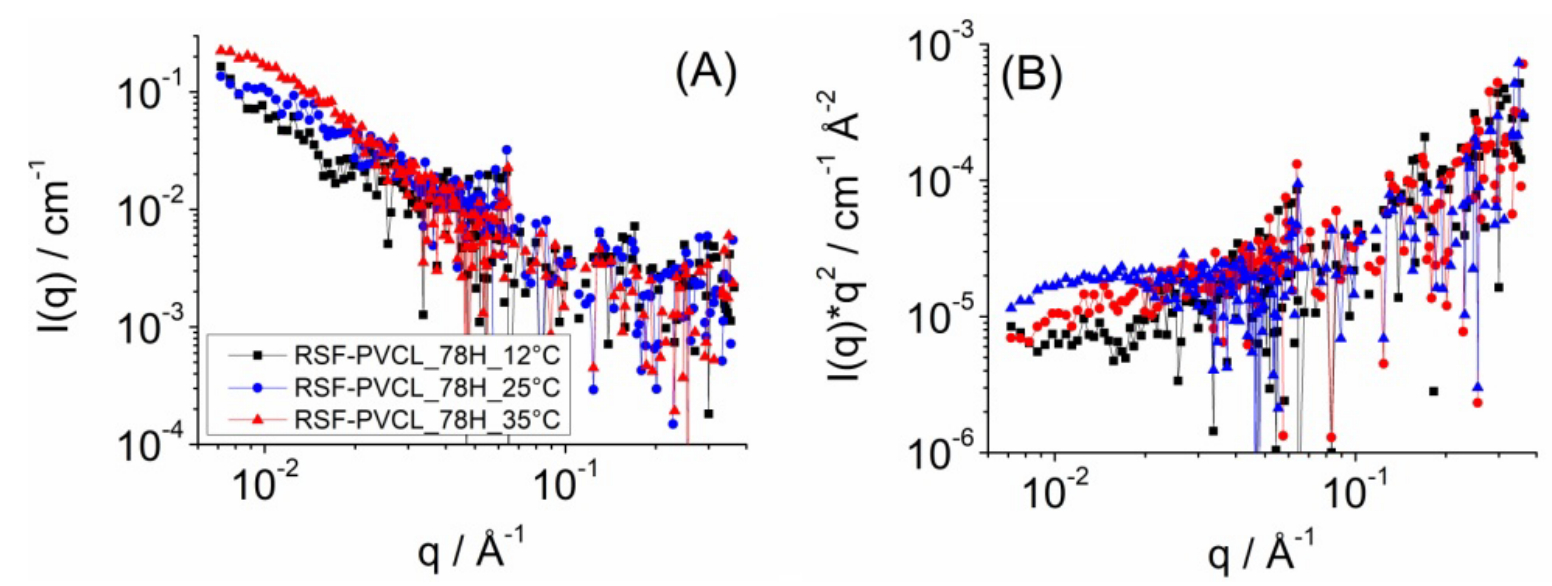

Figure 3. (A) SANS curves and (B) respective Kratky plots of RSF-PVCL blend system as a function of temperature and contrast matched to PVCL.

\section{Supramolecular structure of RSF hydrogels}

Although RSF does not form quick gels with temperature like PVCL, hydrogels of RSF can be formed by aging (physically crosslinked) and photocrosslinking (chemically crosslinked) (7, 39). From our previous experiments, a minimum of $15 \mathrm{wt} \%$ RSF was observed to get a free standing gels by photocrosslinking (7). Figure 4A compares the scattering profile of 15 wt $\%$ RSF in solution, physically crosslinked hydrogel and photochemically crosslinked hydrogel at $25{ }^{\circ} \mathrm{C}$. Compared to $2 \mathrm{wt} \%$, the $15 \mathrm{wt} \% \mathrm{RSF}$ solution showed relatively lower $\mathrm{Rg}$ $(7.51 \pm 0.06)$ as increase in concentration of proteins reduces the available aqueous volume and elevates the osmotic pressure relative to pure water, thereby causing them to adapt to more compact conformations (32). RSF in solution exists in $\alpha$-helix and random coil conformations (soluble form), which upon aging transitions to crystalline $\beta$-sheets and is rendered insoluble (physically crosslinked gel) $(26,29,40)$. On the other hand, in 
photocrosslinked RSF hydrogel, the RSF molecules are crosslinked by covalent dityrosine crosslinks (7). The tyrosine crosslinking is anticipated to form crystalline $\beta$-sheets (due to crosslinking between tyrosine residues in regular sequences of RSF as well as amorphous structures (random coil) in RSF hydrogel (41). Therefore, RSF hydrogels are anticipated to contain hydrophobic domains (predominantly containing $\beta$-sheet) in the system, which can be observed by a Porod slope of $\sim 4$ exhibited by hydrogels (42). A Porod slope of $\sim 4$ is a characteristic of sharp interface of structures formed with the surrounding environment, which in this case can be attributed to $\beta$-sheet structures (crystalline) that exhibit sharp interface with $\mathrm{D}_{2} \mathrm{O}$ and/or amorphous protein matrix $(19,43)$. Further, the hydrophobic domains in the hydrogel system can be clearly seen in Kratky plot with a distinct high-q correlation peak (Figure 4B). The size of hydrophobic domains was estimated to be around 6 $\mathrm{nm}$ from correlation peak q value of $\sim 0.1$ using the relation $\mathrm{d}=2 \pi / q(19)$. On the other hand, the difference in mid-q scattering intensity observed between physically and chemically crosslinked RSF hydrogel can be attributed to their difference in the order of secondary structures. The order of secondary structure in gels affects the voids which inturn influences water uptake capacity. To put it simply, higher the order of secondary structure, higher the crosslink density, lower water uptake, lower mid-q scattering, whereas higher mechanical properties $(13,44)$. Compared to physically crosslinked RSF hydrogel, the photocrosslinked RSF hydrogel demonstrated higher mechanical strength, lower water uptake, lower mid-q scattering intensity and therefore lower the order of secondary structure (13). Moreover, an increased trend in mid-q region intensity observed towards low-q suggests presence of structural assemblies on a range of length scales in the sample, extending beyond the measured SANS range. 

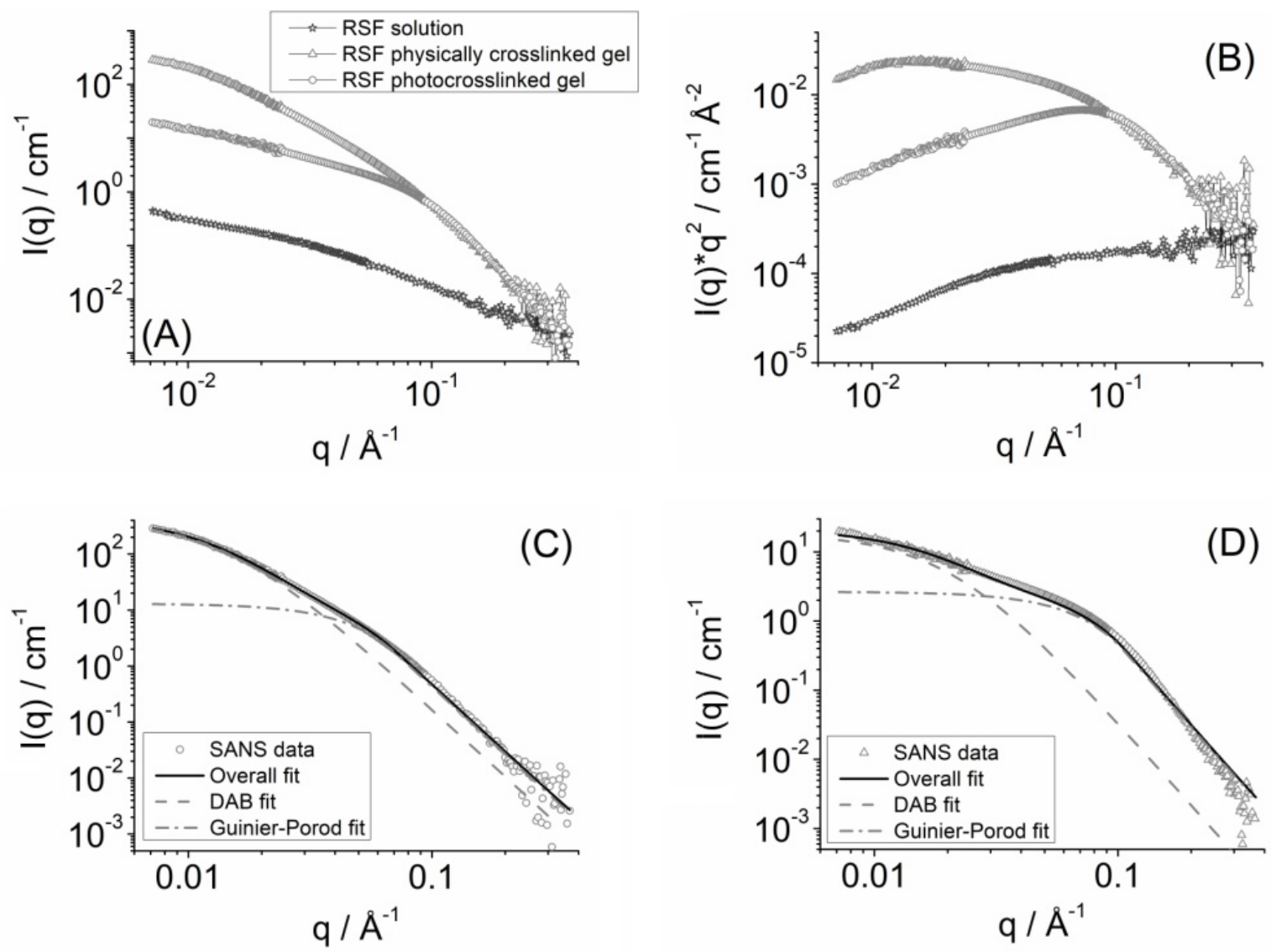

Figure 4. (A) SANS curves and (B) respective Kratky plots of RSF solution and hydrogels.

(C) and (D) are shape-independent model function fits to physically crosslinked and photocrosslinked hydrogels, respectively.

Table 2. Structural parameters of gel samples estimated from SANS data.

\begin{tabular}{lccc}
\hline Sample & \multicolumn{2}{c}{ DAB (mid-q) + Guinier-Porod (high-q) fit } \\
\cline { 2 - 4 } & Correlation length (nm) & Rg (nm) & High- $\boldsymbol{q}$ Porod slope \\
\hline Physically crosslinked RSF hydrogel by aging & $7.14 \pm 0.24$ & $3.49 \pm 0.01$ & $4.00 \pm 0.01$ \\
Photocrosslinked RSF hydrogel & $4.78 \pm 0.36$ & $2.29 \pm 0.01$ & $4.00 \pm 0.01$ \\
Photocrosslinked RSF-PVCL hybrid hydrogel & $18.01 \pm 1.55$ & $2.91 \pm 0.04$ & $3.50 \pm 0.04$ \\
\hline
\end{tabular}

In order to obtain in-depth structural information and to further our understanding of RSF hydrogels the SANS data were fit to shape-independent form factor function (Figure 4C 
and 4D) combining Guinier-Porod model, A(q) at high-q and Debye Anderson Brumberger (DAB) model, B(q) at mid-q, as given in equation 4 (42).

$$
I(q)=A(q)+B(q)
$$

The structural parameters estimated from the fits are given in Table 2. The DAB model calculates the scattering from a randomly distributed two-phase system characterized by a single scale correlation length (L), which is a measure of the average spacing between regions of the two phases (45). The DAB model also assumes smooth interfaces between the two phases and hence exhibits Porod behavior $\left(\mathrm{I} \sim \mathrm{q}^{-4}\right)$ at high-q (45). From Table 2 it can be observed that the average hydrophobic domain size in physically crosslinked RSF hydrogel was estimated to be $\sim 3.5 \mathrm{~nm}$, which is randomly distributed in the amorphous matrix containing hydrophilic domains of silk fibroin with a correlation length (average spacing between $\beta$-sheets) of $\sim 7.1 \mathrm{~nm}$. Photocrosslinked RSF hydrogel demonstrated relatively smaller hydrophobic domain size $(\mathrm{Rg} \sim 2.3 \mathrm{~nm})$ and correlation length $(\sim 4.8 \mathrm{~nm})$ due to restriction in co-localization of tyrosine residues by dityrosine crosslinks, which is anticipated to give rise to its higher mechanical properties $(42,46)$. A schematic of RSF hydrogel nanostructure is given in Figure 5.
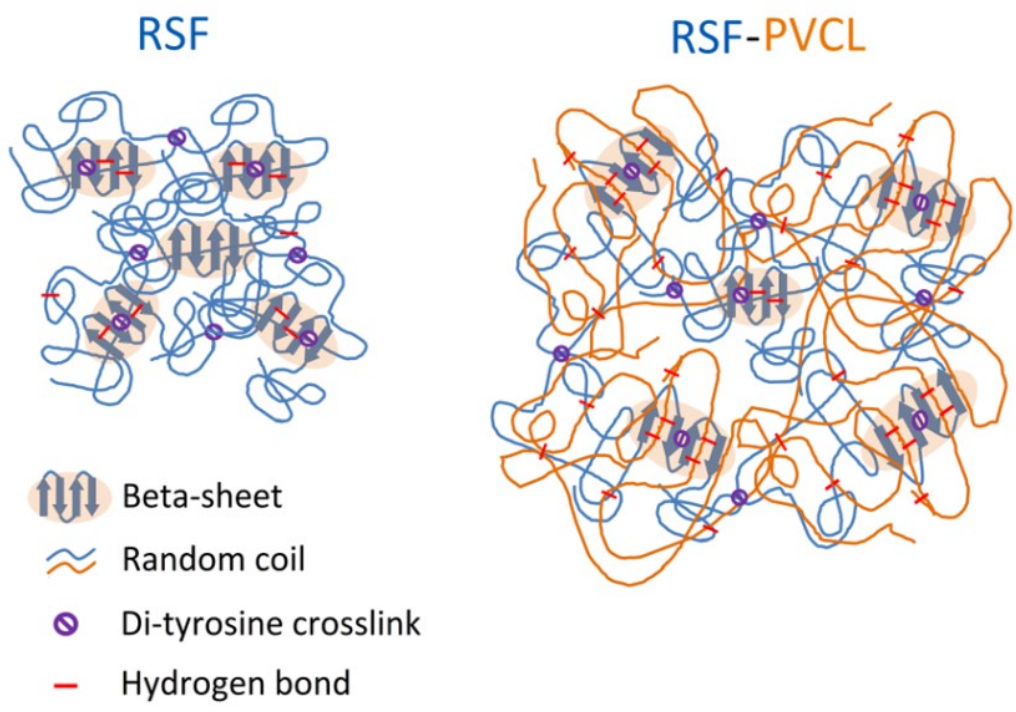

- Hydrogen bond 
Figure 5. A schematic of RSF and RSF-PVCL hybrid hydrogel nanostructure. Reproduced with permission from reference (42). Copyright (2018) Elsevier.

\section{Supramolecular structure of RSF-PVCL hybrid hydrogels}

Applying the photochemical crosslinking method, double network RSF-PVCL hybrid hydrogel with a highly crosslinked rigid phase (RSF) and a physically entrapped soft component (PVCL) have been fabricated (18). Figure 6A compares the scattering profile of physically and photochemically crosslinked RSF-PVCL hybrid hydrogels. It can be observed that the physical hydrogel obtained by equilibrating the solution blends above LCST temperature of PVCL demonstrated collapsed polymer network structure (less ordered secondary structure) with a measured Porod slope of 2.27 , whereas photocrosslinked hydrogel demonstrated partially ordered/crystalline hydrophobic structures with a Porod slope of 3.5 (33). The difference in Porod slope observed between photocrosslinked RSF hydrogel and photocrosslinked RSF-PVCL hybrid hydrogel can be attributed to the increased contribution from the uncollapsed PVCL chains (exhibiting power law behaviour of $\mathrm{q}^{-2}$ ) (42). Further, Kratky plot shows the characteristic of less ordered structure for physical hydrogel in the nanoscale, whereas highly ordered structure for photochemically crosslinked hydrogel with correlation peak observed around q value of $0.08 \AA^{-1}$ (Figure 6B). Therefore, the SANS data of photocroslinked RSF-PVCL hybrid hydrogel was fit (Figure 6C) with shapeindependent form factor function (equation 4), and the obtained structural parameters are given in Table 2. From Table 2 it can be observed that the average hydrophobic domain size and correlation length of photocrosslinked RSF hydrogel showed $26 \%$ and $275 \%$ increase, respectively with PVCL substitution and subsequent photocrosslinking. The physically croslinked RSF-PVCL hybrid hydrogel was not fit with DAB function as it did not show a distinctive hydrophobic and hydrophilic structure. The PVCL forms strong hydrogen bonding with RSF thereby affecting its secondary structural arrangement during crosslinking or 
gelation. This is also in correlation with measured decrease in crosslink density of photocrosslinked RSF hydrogel with PVCL (18). Moreover, an increased trend in mid-q region intensity observed towards low-q suggests presence of structural assemblies on a range of length scales in the sample, extending beyond the measured SANS range. A schematic of RSF-PVCL hybrid hydrogel nanostructure is given in Figure 5.
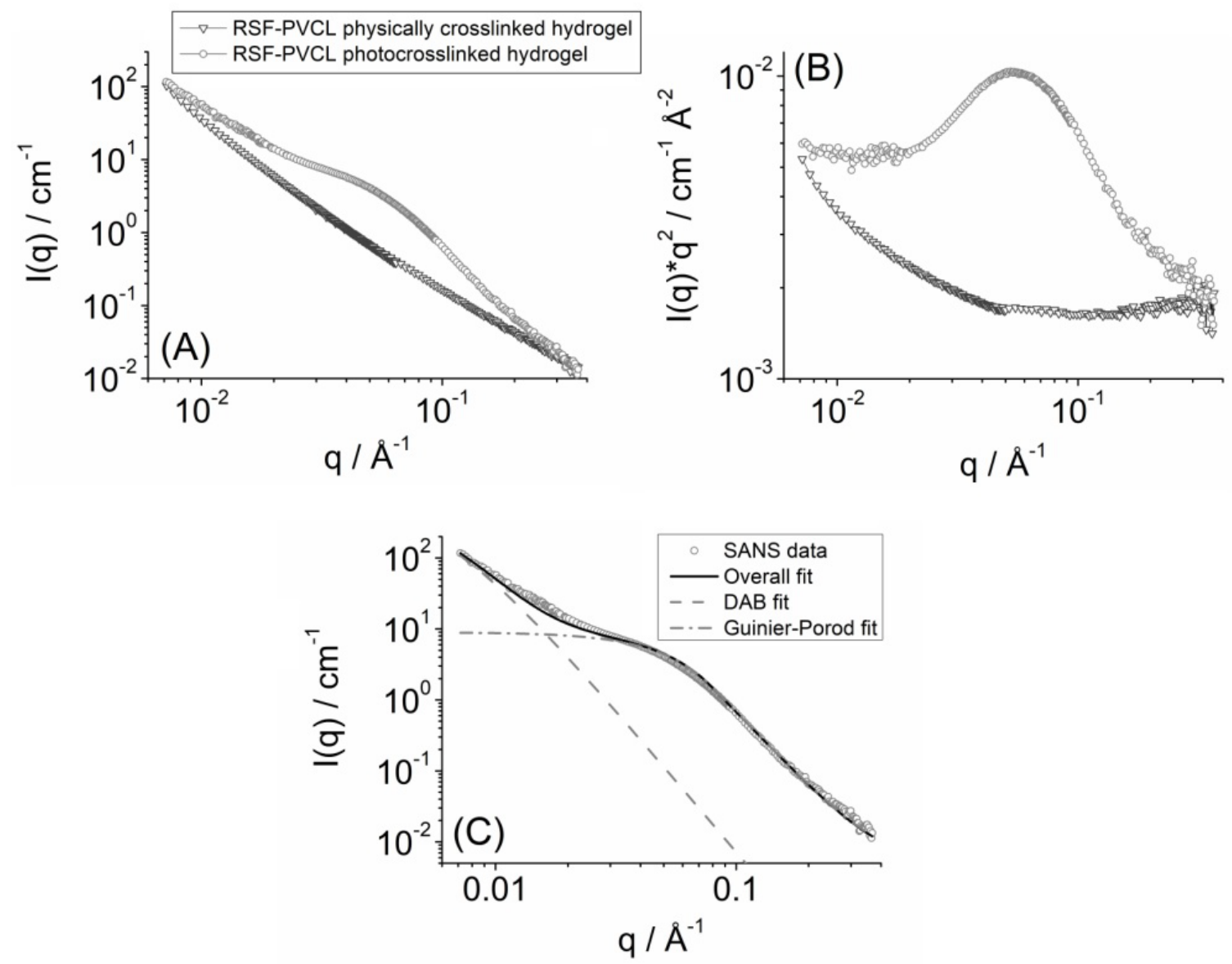

Figure 6. (A) SANS curves and (B) respective Kratky plots of RSF-PVCL hybrid hydrogels.

(C) Shape-independent model function fits to photocrosslinked RSF-PVCL hybrid hydrogel.

\section{Conclusions}

In summary, the evolution of nanostructure of biomimetic hydrogels fabricated using RSF and PVCL solutions has been studies using contrast variation SANS. At $12{ }^{\circ} \mathrm{C}$, PVCL exhibited swollen coil secondary structure in solution, whereas RSF exhibited Gaussian coil 
structure. With increase in temperature, PVCL exhibited swollen coil to Gaussian coil secondary structure change above a transition temperature of $\sim 32{ }^{\circ} \mathrm{C}$ and the solution turned to physical hydrogel. However, no change in secondary structure and solution to gel transition was observed for RSF solution. RSF-PVCL blend solutions exhibited Gausian coil to collapsed coil secondary structure change above $\sim 25{ }^{\circ} \mathrm{C}$ and the solution turned to physical hydrogel. Contrast variation SANS further revealed that the RSF molecules without undergoing any structural change of its own induced/enhanced the intrinsic structural changes of PVCL in RSF-PVCL blend solution, which caused gelation of RSF-PVCL blend solution to occur at relatively lower temperature. Photocrosslinked RSF hydrogel exhibited ordered structures comprising hydrophobic and hydrophobic domains. Photocrosslinking RSF in the presence of PVCL caused decrease in the order of RSF secondary structure in hydrophilic domains, whereas increase in the size of hydrophobic domains. The study provides further insight into the nanostructure of RSF-PVCL hybrid hydrogel systems and benefits in understanding their structure-property relations.

\section{Acknowledgements}

This research has been financially supported by the Australian Research Council (ARC) through Discovery Grant funding (DP160101267). The SANS experiments were supported through an ANSTO beam time award (P3550).

\section{References}

1. Huang, G.; Li, F.; Zhao, X.; Ma, Y.; Li, Y.; Lin, M.; Jin, G.; Lu, T. J.; Genin, G. M.; Xu, F. Functional and Biomimetic Materials for Engineering of the Three-Dimensional Cell Microenvironment. Chem. Rev. 2017, 117, 12764-12850.

2. Gulyuz, U.; Okay, O. Self-Healing Poly(acrylic acid) Hydrogels with Shape Memory Behavior of High Mechanical Strength. Macromolecules 2014, 47, 6889-6899. 
3. Alexandre, E.; Boudjema, K.; Schmitt, B.; Cinqualbre, J.; Jaeck, D.; Lutz, P. J. Poly(Ethylene oxide) Hydrogels: Valuable Candidates for an Artificial Pancreas? In Polymeric Drug Delivery II. ACS Symposium Series 2006, 924, 135-149.

4. Sala, R. L.; Kwon, M. Y.; Kim, M.; Gullbrand, S. E.; Henning, E. A.; Mauck, R. L.; Camargo, E. R.; Burdick, J. A. Thermosensitive Poly(N-vinylcaprolactam) Injectable Hydrogels for Cartilage Tissue Engineering. Tissue Eng., Part A 2017, 23, 935-945.

5. Walters, B. D.; Stegemann, J. P. Strategies for directing the structure and function of three-dimensional collagen biomaterials across length scales. Acta Biomater. 2014, 10, 1488-1501.

6. Rowley, J. A.; Madlambayan, G.; Mooney, D. J. Alginate hydrogels as synthetic extracellular matrix materials. Biomaterials 1999, 20, 45-53.

7. Whittaker, J. L.; Choudhury, N. R.; Dutta, N. K.; Zannettino, A. Facile and rapid ruthenium mediated photo-crosslinking of Bombyx mori silk fibroin. J. Mater. Chem. B 2014, 2, 6259-6270.

8. Cortez-Lemus, N. A.; Licea-Claverie, A. Poly(N-vinylcaprolactam), a comprehensive review on a thermoresponsive polymer becoming popular. Prog. Polym. Sci. 2016, 53, 151 .

9. Kapoor, S.; Kundu, S. C. Silk protein-based hydrogels: Promising advanced materials for biomedical applications. Acta Biomater. 2016, 31, 17-32.

10. Hinkley, J. A.; Morgret, L. D.; Gehrke, S. H. Tensile properties of two responsive hydrogels. Polymer 2004, 45, 8837-8843.

11. Rockwood, D. N.; Preda, R. C.; Yücel, T.; Wang, X.; Lovett, M. L.; Kaplan, D. L. Materials fabrication from Bombyx mori silk fibroin. Nat. Protoc. 2011, 6, 1612.

12. Koh, L.-D.; Cheng, Y.; Teng, C.-P.; Khin, Y.-W.; Loh, X.-J.; Tee, S.-Y.; Low, M.; Ye, E.; Yu, H.-D.; Zhang, Y.-W.; Han, M.-Y. Structures, mechanical properties and applications of silk fibroin materials. Prog. Polym. Sci. 2015, 46, 86-110.

13. Whittaker, J. L. Engineered silk-based biomimetic hydrogels for cell growth. University of South Australia, 2016.

14. Balu, R.; Whittaker, J.; Dutta, N. K.; Elvin, C. M.; Choudhury, N. R. Multi-responsive biomaterials and nanobioconjugates from resilin-like protein polymers. J. Mater. Chem. B 2014, 2, 5936-5947.

15. Maeda, Y.; Nakamura, T.; Ikeda, I. Hydration and Phase Behavior of Poly(Nvinylcaprolactam) and Poly(N-vinylpyrrolidone) in Water. Macromolecules 2002, 35, 217-222. 
16. Kozlovskaya, V.; Baggett, J.; Godin, B.; Liu, X.; Kharlampieva, E. Hydrogen-Bonded Multilayers of Silk Fibroin: From Coatings to Cell-Mimicking Shaped Microcontainers. ACS Macro Lett. 2012, 1, 384-387.

17. Espinosa-Dzib, A.; Chen, J.; Zavgorodnya, O.; Kozlovskaya, V.; Liang, X.; Kharlampieva, E. Tuning assembly and enzymatic degradation of silk/poly(Nvinylcaprolactam) multilayers via molecular weight and hydrophobicity. Soft Matter 2015, 11, 5133-5145.

18. Whittaker, J. L.; Dutta, N. K.; Zannettino, A.; Choudhury, N. R. Engineering DN hydrogels from regenerated silk fibroin and poly(N-vinylcaprolactam). J. Mater. Chem. $B$ 2016, 4, 5519-5533.

19. Feigin, L. A.; Svergun, D. I. Structure Analysis by Small-Angle X-Ray and Neutron Scattering. Springer US: 2013.

20. Balu, R.; Mata, J. P.; Knott, R.; Elvin, C. M.; Hill, A. J.; Choudhury, N. R.; Dutta, N. K. Effects of Crowding and Environment on the Evolution of Conformational Ensembles of the Multi-Stimuli-Responsive Intrinsically Disordered Protein, Rec1-Resilin: A SmallAngle Scattering Investigation. J. Phys. Chem. B 2016, 120, 6490-6503.

21. Shibayama, M. Small-angle neutron scattering on polymer gels: phase behavior, inhomogeneities and deformation mechanisms. Polym. J. 2010, 43, 18.

22. Heller, W. Small-angle neutron scattering and contrast variation: a powerful combination for studying biological structures. Acta Crystallogr., Sect. D 2010, 66, 1213-1217.

23. Wood, K.; Mata, J. P.; Garvey, C. J.; Wu, C.-M.; Hamilton, W. A.; Abbeywick, P.; Bartlett, D.; Bartsch, F.; Baxter, P.; Booth, N.; Brown, W.; Christoforidis, J.; Clowes, D.; d'Adam, T.; Darmann, F.; Deura, M.; Harrison, S.; Hauser, N.; Horton, G.; Federici, D.; Franceschini, F.; Hanson, P.; Imamovic, E.; Imperia, P.; Jones, M.; Kennedy, S.; Kim, S.; Lam, T.; Lee, W. T.; Lesha, M.; Mannicke, D.; Noakes, T.; Olsen, S. R.; Osborn, J. C.; Penny, D.; Perry, M.; Pullen, S. A.; Robinson, R. A.; Schulz, J. C.; Xiong, N.; Gilbert, E. P. QUOKKA, the pinhole small-angle neutron scattering instrument at the OPAL Research Reactor, Australia: design, performance, operation and scientific highlights. J. Appl. Cryst. 2018, 51, 294-314.

24. Lipfert, J.; Doniach, S. Small-Angle X-Ray Scattering from RNA, Proteins, and Protein Complexes. Annu. Rev. Biophys. Biomol. Struct. 2007, 36, 307-327.

25. Jayawardane, D.; Pan, F.; Lu, J. R.; Zhao, X. Interfacial Adsorption of Silk Fibroin Peptides and Their Interaction with Surfactants at the Solid-Water Interface. Langmuir 2016, 32, 8202-8211. 
26. Schneider, F.; Balaceanu, A.; Feoktystov, A.; Pipich, V.; Wu, Y.; Allgaier, J.; PyckhoutHintzen, W.; Pich, A.; Schneider, G. J. Monitoring the Internal Structure of Poly(Nvinylcaprolactam) Microgels with Variable Cross-Link Concentration. Langmuir 2014, 30, 15317-15326.

27. Burger, C.; Ruland, W. Evaluation of equatorial orientation distributions. J. Appl. Crystallogr. 2006, 39, 889-891.

28. Konarev, P. V.; Volkov, V. V.; Sokolova, A. V.; Koch, M. H. J.; Svergun, D. I. PRIMUS: a Windows PC-based system for small-angle scattering data analysis. J. Appl. Crystallogr. 2003, 36, 1277-1282.

29. Saffer, E. M.; Lackey, M. A.; Griffin, D. M.; Kishore, S.; Tew, G. N.; Bhatia, S. R. SANS study of highly resilient poly(ethylene glycol) hydrogels. Soft Matter 2014, 10, 1905-1916.

30. Putnam, C. D.; Hammel, M.; Hura, G. L.; Tainer, J. A. X-ray solution scattering (SAXS) combined with crystallography and computation: defining accurate macromolecular structures, conformations and assemblies in solution. Q. Rev. Biophys. 2007, 40, 191285.

31. Lebedev, V. T.; Török, G.; Cser, L.; Kali, G.; Kirsh, Y. E.; Sibilev, A. I.; Orlova, D. N., NSE-study of poly(N-vinylcaprolactam) by coil-globule transition. Phys. B: Condens. Matter. 2001, 297, 50-54.

32. Small Angle X-Ray and Neutron Scattering from Solutions of Biological Macromolecules; Svergun, D. I.; Koch, M. H. J.; Timmins, P. A.; May, R. P. , Eds.; Oxford University Press: Oxford, UK, 2013.

33. Small Angle X-ray Scattering; Glatter, O.; Kratky, O., Eds.; Academic Press: London, UK, 1982.

34. Veronique, R.-B.; Dominique, D. How Random are Intrinsically Disordered Proteins? A Small Angle Scattering Perspective. Curr. Protein Pept. Sci. 2012, 13, 55-75.

35. Bernadó, P. Effect of interdomain dynamics on the structure determination of modular proteins by small-angle scattering. Eur. Biophys. J. 2010, 39, 769-780.

36. Hammouda, B. SANS from homogeneous polymer mixtures: A unified overview. In Polymer Characteristics, Springer Berlin Heidelberg: Berlin, Heidelberg, 1993.

37. Benoit, H. La Diffusion de La Lumiere par des Macromolecules en Chaines en Solution dans un Bon Solvant. Comptes Rendus 1957, 245, 2244-2247.

38. Hammouda, B. A new Guinier-Porod model. J. Appl. Crystallogr. 2010, 43, 716-719. 
39. Zainuddin; Le, T. T.; Park, Y.; Chirila, T. V.; Halley, P. J.; Whittaker, A. K. The behavior of aged regenerated Bombyx mori silk fibroin solutions studied by $1 \mathrm{H}$ NMR and rheology. Biomaterials 2008, 29, 4268-4274.

40. Polymeric Hydrogels as Smart Biomaterials; Kalia, S., Ed.; Springer International Publishing: Cham, SZ, 2015.

41. Ha, S.-W.; Gracz, H. S.; Tonelli, A. E.; Hudson, S. M. Structural Study of Irregular Amino Acid Sequences in the Heavy Chain of Bombyx mori Silk Fibroin. Biomacromolecules 2005, 6, 2563-2569.

42. Whittaker, J. L.; Balu, R.; Knott, R.; de-Campo, L.; Mata, J. P.; Rehm, C.; Hill, A. J.; Dutta, N. K.; Choudhury, N. R. Structural evolution of photocrosslinked silk fibroin and silk fibroin-based hybrid hydrogels: A small angle and ultra-small angle scattering investigation. Int. J. Biol. Macromol. 2018 (Article in press, DOI: 10.1016/j.ijbiomac.2018.03.044).

43. Drnovsek, N.; Kocen, R.; Gantar, A.; Drobnic-Kosorok, M.; Leonardi, A.; Krizaj, I.; Recnik, A.; Novak, S. Size of silk fibroin $\beta$-sheet domains affected by $\mathrm{Ca}^{2+}$. J. Mater. Chem. B 2016, 4, 6597-6608.

44. Lawrence, B. D.; Wharram, S.; Kluge, J. A.; Leisk, G. G.; Omenetto, F. G.; Rosenblatt, M. I.; Kaplan, D. L. Effect of Hydration on Silk Film Material Properties. Macromol. Biosci. 2010, 10, 393-403.

45. Debye, P.; Anderson, H. R.; Brumberger, H. Scattering by an inhomogeneous solid. II. The correlation function and its application, J. Appl. Phys. 1957, 28, 679.

46. Su, D.; Yao, M.; Liu, J.; Zhong, Y.; Chen, X.; Shao, Z. Enhancing mechanical properties of silk fibroin hydrogel through restricting the growth of $\beta$-Sheet domains. ACS Appl. Mater. Interf. 2017, 9, 17489-17498. 